Supporting information

\title{
Iron modulation of copper uptake and toxicity in a green alga (Chlamydomonas reinhardtii)
}

\author{
Authors: \\ Emeric Kochoni and Claude Fortin* \\ Institut national de la Recherche scientifique, Centre Eau Terre Environnement, 490 de la \\ Couronne, Québec, QC, G1K 9A9, Canada \\ *corresponding author: claude.fortin@ete.inrs.ca
}

12 Tables and 1 Figure 
Tables

Table S1: Ionic composition of MHSM1 and LM1 to LM4 media. Free ion concentrations were calculated using MINEQL+ 5.0. All concentrations are given in molar (M).

\begin{tabular}{|c|c|c|c|c|c|c|c|c|c|c|}
\hline \multicolumn{2}{|c|}{ Media } & AHSM1 & \multicolumn{2}{|c|}{ LM1 } & \multicolumn{2}{|l|}{ LM2 } & \multicolumn{2}{|l|}{ LM3 } & \multicolumn{2}{|c|}{ LM4 } \\
\hline Compound & Total & Free & Total & Free & Total & Free & Total & Free & Total & Free \\
\hline $\mathrm{BO}_{3}$ & $3.01 \mathrm{E}-06$ & $2.99 \mathrm{E}-06$ & $3.01 \mathrm{E}-06$ & $2.99 \mathrm{E}-06$ & $3.01 \mathrm{E}-06$ & $2.99 \mathrm{E}-06$ & $3.01 \mathrm{E}-06$ & $2.99 \mathrm{E}-06$ & $3.01 \mathrm{E}-06$ & $2.99 \mathrm{E}-06$ \\
\hline $\mathrm{Ca}$ & $6.80 \mathrm{E}-05$ & $6.57 \mathrm{E}-05$ & $3.28 \mathrm{E}-05$ & 2.03E-05 & $3.28 \mathrm{E}-05$ & $2.03 E-05$ & $3.28 \mathrm{E}-05$ & $2.03 E-05$ & $3.28 \mathrm{E}-05$ & 2.03E-05 \\
\hline $\mathrm{Cl}$ & 5.98E-06 & 5.97E-06 & 5.00E-06 & 4.97E-06 & 5.00E-06 & 4.97E-06 & $5.00 \mathrm{E}-06$ & 4.97E-06 & 5.00E-06 & 4.97E-06 \\
\hline $\mathrm{CO}_{3}$ & Atm & $3.20 \mathrm{E}-08$ & Atm & $3.95 \mathrm{E}-08$ & Atm & $3.95 \mathrm{E}-08$ & Atm & $3.95 \mathrm{E}-08$ & Atm & $3.95 \mathrm{E}-08$ \\
\hline Co & $1.09 \mathrm{E}-08$ & 3.59E-09 & $6.79 \mathrm{E}-08$ & $1.04 \mathrm{E}-11$ & $6.79 \mathrm{E}-08$ & $1.04 \mathrm{E}-11$ & $6.79 \mathrm{E}-08$ & $1.04 \mathrm{E}-11$ & $6.79 \mathrm{E}-08$ & $1.04 \mathrm{E}-11$ \\
\hline $\mathrm{Cu}$ & 7.04E-08 & $1.49 \mathrm{E}-10$ & 3.69E-08 & $1.04 \mathrm{E}-14$ & $3.74 \mathrm{E}-07$ & $1.04 \mathrm{E}-13$ & $3.74 \mathrm{E}-06$ & $1.04 \mathrm{E}-12$ & 3.74E-05 & $1.04 \mathrm{E}-11$ \\
\hline $\mathrm{Fe}$ & $5.92 \mathrm{E}-07$ & $2.25 \mathrm{E}-18$ & $1.28 \mathrm{E}-07$ & 1.07E-19 & $1.28 \mathrm{E}-07$ & 1.07E-19 & $1.28 \mathrm{E}-07$ & $1.07 E-19$ & $1.28 \mathrm{E}-07$ & 1.07E-19 \\
\hline K & $4.22 \mathrm{E}-03$ & $4.21 \mathrm{E}-03$ & $4.72 \mathrm{E}-03$ & 4.68E-03 & $4.72 \mathrm{E}-03$ & 4.68E-03 & $4.72 \mathrm{E}-03$ & $4.68 \mathrm{E}-03$ & 4.72E-03 & $4.68 \mathrm{E}-03$ \\
\hline $\mathrm{Mg}$ & $8.12 \mathrm{E}-05$ & $7.89 E-05$ & $8.12 \mathrm{E}-05$ & 7.63E-05 & $8.12 \mathrm{E}-05$ & 7.63E-05 & $8.12 \mathrm{E}-05$ & $7.63 \mathrm{E}-05$ & $8.12 \mathrm{E}-05$ & 7.63E-05 \\
\hline $\mathrm{Mn}$ & $2.10 \mathrm{E}-06$ & $1.86 \mathrm{E}-06$ & $3.28 \mathrm{E}-08$ & 5.15E-09 & $3.28 \mathrm{E}-08$ & 5.15E-09 & $3.28 \mathrm{E}-08$ & 5.15E-09 & $3.28 \mathrm{E}-08$ & 5.15E-09 \\
\hline $\mathrm{MoO}_{4}$ & $3.00 \mathrm{E}-08$ & $3.00 \mathrm{E}-08$ & $3.00 \mathrm{E}-08$ & $2.95 \mathrm{E}-08$ & $3.00 \mathrm{E}-08$ & $2.95 \mathrm{E}-08$ & $3.00 \mathrm{E}-08$ & $2.95 \mathrm{E}-08$ & $3.00 \mathrm{E}-08$ & $2.95 \mathrm{E}-08$ \\
\hline $\mathrm{Na}$ & $1.02 \mathrm{E}-04$ & $1.02 \mathrm{E}-04$ & 1.67E-02 & 1.66E-02 & 1.67E-02 & $1.66 \mathrm{E}-02$ & $1.67 \mathrm{E}-02$ & $1.66 \mathrm{E}-02$ & 1.67E-02 & $1.66 \mathrm{E}-02$ \\
\hline $\mathrm{NH}_{4}$ & $9.37 \mathrm{E}-04$ & $9.32 \mathrm{E}-04$ & $9.37 \mathrm{E}-04$ & $9.32 \mathrm{E}-04$ & 9.37E-04 & $9.32 \mathrm{E}-04$ & 9.37E-04 & $9.32 \mathrm{E}-04$ & $9.37 \mathrm{E}-04$ & $9.32 \mathrm{E}-04$ \\
\hline $\mathrm{NO}_{3}$ & 5.07E-03 & $5.06 \mathrm{E}-03$ & 1.60E-02 & $1.59 \mathrm{E}-02$ & $1.60 \mathrm{E}-02$ & $1.59 \mathrm{E}-02$ & $1.60 \mathrm{E}-02$ & $1.59 \mathrm{E}-02$ & 1.60E-02 & $1.59 \mathrm{E}-02$ \\
\hline $\mathrm{PO}_{4}$ & $1.37 \mathrm{E}-04$ & $4.06 \mathrm{E}-10$ & $1.37 \mathrm{E}-04$ & $5.27 \mathrm{E}-10$ & $1.37 \mathrm{E}-04$ & $5.27 \mathrm{E}-10$ & $1.37 \mathrm{E}-04$ & $5.27 \mathrm{E}-10$ & $1.37 \mathrm{E}-04$ & $5.27 \mathrm{E}-10$ \\
\hline $\mathrm{SO}_{4}$ & $8.12 \mathrm{E}-05$ & $7.80 \mathrm{E}-05$ & $8.12 E-05$ & $7.52 \mathrm{E}-05$ & $8.12 \mathrm{E}-05$ & $7.52 \mathrm{E}-05$ & $8.12 \mathrm{E}-05$ & $7.52 \mathrm{E}-05$ & $8.12 \mathrm{E}-05$ & $7.52 \mathrm{E}-05$ \\
\hline $\mathrm{Zn}$ & $2.33 \mathrm{E}-07$ & $9.56 \mathrm{E}-08$ & 8.61E-08 & $1.04 \mathrm{E}-11$ & $8.61 \mathrm{E}-08$ & $1.04 \mathrm{E}-11$ & $8.61 \mathrm{E}-08$ & $1.04 \mathrm{E}-11$ & 8.61E-08 & $1.04 \mathrm{E}-11$ \\
\hline EDTA & 8.06E-07 & $6.07 E-18$ & & & & & & & & \\
\hline NTA & & & $1.00 \mathrm{E}-04$ & $8.12 \mathrm{E}-08$ & $1.00 \mathrm{E}-04$ & $8.12 \mathrm{E}-08$ & $1.04 \mathrm{E}-04$ & $8.12 \mathrm{E}-08$ & $1.36 \mathrm{E}-04$ & $8.12 \mathrm{E}-08$ \\
\hline MOPS & $1.00 \mathrm{E}-02$ & 4.18E-03 & $1.00 \mathrm{E}-02$ & 4.31E-03 & $1.00 \mathrm{E}-02$ & 4.31E-03 & $1.00 \mathrm{E}-02$ & $4.31 \mathrm{E}-03$ & $1.00 \mathrm{E}-02$ & 4.31E-03 \\
\hline
\end{tabular}


Table S2: Components and proportions of the stock solutions used in the preparation of the MHSM1 culture medium

\begin{tabular}{|c|c|c|c|c|}
\hline Stock solutions $^{\mathrm{a}}$ & Volume used $^{\mathrm{b}}$ & Components & Concentrations & Suppliers \\
\hline \multirow{3}{*}{ Ammonium \#2 } & \multirow{3}{*}{$5 \mathrm{~mL} \cdot \mathrm{L}^{-1}$} & $\mathrm{NH}_{4} \mathrm{NO}_{3}$ & $15.0 \mathrm{~g} \cdot \mathrm{L}^{-1}$ & Omega \\
\hline & & $\mathrm{MgSO}_{4}, 7 \mathrm{H}_{2} \mathrm{O}$ & $4.00 \mathrm{~g} \cdot \mathrm{L}^{-1}$ & Omega \\
\hline & & $\mathrm{Ca}\left(\mathrm{NO}_{3}\right)_{2}, 4 \mathrm{H}_{2} \mathrm{O}$ & $3.21 \mathrm{~g} \cdot \mathrm{L}^{-1}$ & $\mathrm{BDH}$ \\
\hline \multirow{2}{*}{ Phosphate \#2 } & \multirow{2}{*}{$250 \mu \mathrm{L} \cdot \mathrm{L}^{-1}$} & $\mathrm{KH}_{2} \mathrm{PO}_{4}$ & $29.6 \mathrm{~g} \cdot \mathrm{L}^{-1}$ & $\mathrm{BDH}$ \\
\hline & & $\mathrm{K}_{2} \mathrm{HPO}_{4}$ & $57.6 \mathrm{~g} \cdot \mathrm{L}^{-1}$ & $\mathrm{BDH}$ \\
\hline $\mathrm{KNO}_{3}(1.0 \mathrm{M})$ & $4 \mathrm{~mL} \cdot \mathrm{L}^{-1}$ & & $101 \mathrm{~g} \cdot \mathrm{L}^{-1}$ & Omega \\
\hline \multirow[t]{8}{*}{$\operatorname{AAP}^{d}$} & $1 \mathrm{~mL} \cdot \mathrm{L}^{-1}$ & $\mathrm{H}_{3} \mathrm{BO}_{3}$ & $186 \mathrm{mg} \cdot \mathrm{L}^{-1}$ & $\mathrm{ACP}$ \\
\hline & & $\mathrm{MnCl}_{2}, 4 \mathrm{H}_{2} \mathrm{O}$ & $415 \mathrm{mg} \cdot \mathrm{L}^{-1}$ & Fisher \\
\hline & & $\mathrm{FeCl}_{3}, 6 \mathrm{H}_{2} \mathrm{O}$ & $160 \mathrm{mg} \cdot \mathrm{L}^{-1}$ & $\mathrm{BDH}$ \\
\hline & & $\mathrm{Na}_{2}$ EDTA, $2 \mathrm{H}_{2} \mathrm{O}$ & $300 \mathrm{mg} \cdot \mathrm{L}^{-1}$ & Fisher \\
\hline & & $\mathrm{Zn}^{\mathrm{c}}\left(1 \mathrm{~g} \cdot \mathrm{L}^{-1}\right)$ & $1.59 \mathrm{mg} \cdot \mathrm{L}^{-1}$ & Fisher \\
\hline & & $\operatorname{Co}^{c}\left(1 \mathrm{~g} \cdot \mathrm{L}^{-1}\right)$ & $0.64 \mathrm{mg} \cdot \mathrm{L}^{-1}$ & Fisher \\
\hline & & $\operatorname{Mo}^{c}\left(1 \mathrm{~g} \cdot \mathrm{L}^{-1}\right)$ & $2.88 \mathrm{mg} \cdot \mathrm{L}^{-1}$ & Fisher \\
\hline & & $\mathrm{Cu}^{\mathrm{c}}\left(1 \mathrm{~g} \cdot \mathrm{L}^{-1}\right)$ & $4.47 \mu \mathrm{g} \cdot \mathrm{L}^{-1}$ & Fisher \\
\hline $\mathrm{NaOH}(0.1 \mathrm{M})^{\mathrm{d}}$ & Variable & & $4.00 \mathrm{~g} \cdot \mathrm{L}^{-1}$ & $\mathrm{BDH}$ \\
\hline
\end{tabular}

a: Each solution is filtered on $0.2 \mu \mathrm{m}$ porosity polycarbonate filter membranes.

b: Volume of stock solution needed to prepare the culture medium; final volume $=1000 \mathrm{~mL}$.

c: A standard stock solution for instrument calibration acidified with $\mathrm{HNO}_{3}$.

$\mathrm{d}$ : Solution to be added $24 \mathrm{~h}$ after autoclaving. The amount to be added depends on the presence or absence of buffer and the desired $\mathrm{pH}$. In the absence of buffer, $1 \mathrm{~mL} \cdot \mathrm{L}^{-1}$ is sufficient to reach a $\mathrm{pH}$ of 7 . 
Table S3: Ionic composition of LZn1 to LZn4 media. Free ion concentrations were calculated using MINEQL+ 5.0. All concentrations are given in molar (M).

\begin{tabular}{|c|c|c|c|c|c|c|c|c|}
\hline \multirow{2}{*}{$\begin{array}{r}\text { Media } \\
\text { Compound }\end{array}$} & \multicolumn{2}{|c|}{ LZn1 } & \multicolumn{2}{|c|}{ LZn2 } & \multicolumn{2}{|c|}{ LZn3 } & \multicolumn{2}{|c|}{ LZn4 } \\
\hline & Total & Free & Total & Free & Total & Free & Total & Free \\
\hline $\mathrm{BO}_{3}$ & $3.01 \mathrm{E}-06$ & $2.99 \mathrm{E}-06$ & $3.01 \mathrm{E}-06$ & $2.99 \mathrm{E}-06$ & $3.01 \mathrm{E}-06$ & $2.99 \mathrm{E}-06$ & $3.01 \mathrm{E}-06$ & $2.99 \mathrm{E}-06$ \\
\hline $\mathrm{Ca}$ & $1.06 \mathrm{E}-04$ & 7.67E-05 & $1.06 \mathrm{E}-04$ & 7.67E-05 & 1.06E-04 & 7.67E-05 & $1.06 \mathrm{E}-04$ & 7.67E-05 \\
\hline $\mathrm{Cl}$ & $5.00 \mathrm{E}-06$ & 4.97E-06 & $5.00 \mathrm{E}-06$ & 4.97E-06 & $5.00 \mathrm{E}-06$ & 4.97E-06 & $5.00 \mathrm{E}-06$ & $4.97 \mathrm{E}-06$ \\
\hline $\mathrm{CO}_{3}$ & Atm & $3.95 \mathrm{E}-08$ & Atm & $3.95 \mathrm{E}-08$ & Atm & $3.95 \mathrm{E}-08$ & Atm & $3.95 \mathrm{E}-08$ \\
\hline Co & $1.41 \mathrm{E}-05$ & 3.63E-09 & $1.41 \mathrm{E}-05$ & 3.63E-09 & $1.41 \mathrm{E}-05$ & 3.63E-09 & $1.41 \mathrm{E}-05$ & $3.63 \mathrm{E}-09$ \\
\hline $\mathrm{Cu}$ & $2.21 \mathrm{E}-08$ & $1.05 \mathrm{E}-14$ & $2.21 \mathrm{E}-07$ & $1.05 \mathrm{E}-13$ & $2.21 \mathrm{E}-06$ & $1.05 \mathrm{E}-12$ & $2.21 \mathrm{E}-05$ & $1.05 \mathrm{E}-11$ \\
\hline $\mathrm{Fe}$ & $1.57 \mathrm{E}-06$ & $2.25 \mathrm{E}-18$ & $1.57 \mathrm{E}-06$ & $2.25 \mathrm{E}-18$ & 1.57E-06 & $2.25 \mathrm{E}-18$ & $1.57 \mathrm{E}-06$ & $2.25 \mathrm{E}-18$ \\
\hline K & $4.72 \mathrm{E}-03$ & $4.68 \mathrm{E}-03$ & $4.72 \mathrm{E}-03$ & $4.68 \mathrm{E}-03$ & $4.72 \mathrm{E}-03$ & $4.68 \mathrm{E}-03$ & $4.72 \mathrm{E}-03$ & $4.68 \mathrm{E}-03$ \\
\hline $\mathrm{Mg}$ & 8.39E-05 & $8.02 \mathrm{E}-05$ & 8.39E-05 & $8.02 \mathrm{E}-05$ & 8.39E-05 & $8.02 \mathrm{E}-05$ & 8.39E-05 & $8.02 \mathrm{E}-05$ \\
\hline $\mathrm{Mn}$ & $7.86 \mathrm{E}-06$ & $1.86 \mathrm{E}-06$ & $7.86 \mathrm{E}-06$ & $1.86 \mathrm{E}-06$ & $7.86 \mathrm{E}-06$ & $1.86 \mathrm{E}-06$ & 7.86E-06 & $1.86 \mathrm{E}-06$ \\
\hline $\mathrm{MoO}_{4}$ & $3.00 \mathrm{E}-08$ & 2.97E-08 & 3.00E-08 & 2.97E-08 & 3.00E-08 & 2.97E-08 & $3.00 \mathrm{E}-08$ & $2.97 \mathrm{E}-08$ \\
\hline $\mathrm{Na}$ & 1.67E-02 & $1.66 \mathrm{E}-02$ & 1.67E-02 & 1.66E-02 & 1.67E-02 & 1.66E-02 & 1.67E-02 & $1.66 \mathrm{E}-02$ \\
\hline $\mathrm{NH}_{4}$ & $9.37 \mathrm{E}-04$ & $9.32 \mathrm{E}-04$ & $9.37 \mathrm{E}-04$ & $9.32 \mathrm{E}-04$ & $9.37 \mathrm{E}-04$ & $9.32 \mathrm{E}-04$ & $9.37 \mathrm{E}-04$ & $9.32 \mathrm{E}-04$ \\
\hline $\mathrm{NO}_{3}$ & $1.60 \mathrm{E}-02$ & 1.59E-02 & $1.60 \mathrm{E}-02$ & 1.59E-02 & 1.60E-02 & 1.59E-02 & 1.60E-02 & $1.59 \mathrm{E}-02$ \\
\hline $\mathrm{PO}_{4}$ & 1.37E-04 & $5.25 \mathrm{E}-10$ & 1.37E-04 & $5.25 \mathrm{E}-10$ & 1.37E-04 & $5.25 \mathrm{E}-10$ & $1.37 \mathrm{E}-04$ & $5.25 \mathrm{E}-10$ \\
\hline $\mathrm{SO}_{4}$ & $8.12 \mathrm{E}-05$ & $7.49 \mathrm{E}-05$ & $8.12 \mathrm{E}-05$ & $7.49 \mathrm{E}-05$ & $8.12 E-05$ & 7.49E-05 & $8.12 \mathrm{E}-05$ & $7.49 \mathrm{E}-05$ \\
\hline $\mathrm{Zn}$ & 8.17E-08 & $1.05 \mathrm{E}-11$ & 8.17E-08 & $1.05 \mathrm{E}-11$ & 8.17E-08 & $1.05 \mathrm{E}-11$ & $8.17 \mathrm{E}-08$ & $1.05 \mathrm{E}-11$ \\
\hline NTA & $1.00 \mathrm{E}-04$ & $4.82 \mathrm{E}-08$ & $1.00 \mathrm{E}-04$ & $4.82 \mathrm{E}-08$ & 1.00E-04 & $4.82 \mathrm{E}-08$ & $1.21 \mathrm{E}-04$ & $4.82 \mathrm{E}-08$ \\
\hline MOPS & $1.00 \mathrm{E}-02$ & 4.31E-03 & $1.00 \mathrm{E}-02$ & 4.31E-03 & $1.00 \mathrm{E}-02$ & 4.31E-03 & $1.00 \mathrm{E}-02$ & $4.31 \mathrm{E}-03$ \\
\hline
\end{tabular}


Table S4: Ionic composition of LMn1 to LMn4 media. Free ion concentrations were calculated using MINEQL+ 5.0. All concentrations are given in molar (M).

\begin{tabular}{|c|c|c|c|c|c|c|c|c|}
\hline \multirow{2}{*}{$\begin{array}{l}\text { Media } \\
\text { Compound }\end{array}$} & \multicolumn{2}{|c|}{ LMn1 } & \multicolumn{2}{|c|}{ LMn2 } & \multicolumn{2}{|c|}{ LMn3 } & \multicolumn{2}{|c|}{ LMn4 } \\
\hline & Total & Free & Total & Free & Total & Free & Total & Free \\
\hline $\mathrm{BO}_{3}$ & $3.01 \mathrm{E}-06$ & $2.99 \mathrm{E}-06$ & $3.01 \mathrm{E}-06$ & $2.99 \mathrm{E}-06$ & $3.01 \mathrm{E}-06$ & $2.99 \mathrm{E}-06$ & $3.01 \mathrm{E}-06$ & $2.99 \mathrm{E}-06$ \\
\hline $\mathrm{Ca}$ & $1.06 \mathrm{E}-04$ & $9.61 \mathrm{E}-05$ & $1.06 \mathrm{E}-04$ & $9.61 \mathrm{E}-05$ & $1.06 \mathrm{E}-04$ & $9.61 \mathrm{E}-05$ & $1.06 \mathrm{E}-04$ & $9.61 \mathrm{E}-05$ \\
\hline $\mathrm{Cl}$ & $5.00 \mathrm{E}-06$ & 4.97E-06 & $5.00 \mathrm{E}-06$ & 4.97E-06 & $5.00 \mathrm{E}-06$ & 4.97E-06 & $5.00 \mathrm{E}-06$ & 4.97E-06 \\
\hline $\mathrm{CO}_{3}$ & Atm & $3.95 \mathrm{E}-08$ & Atm & $3.95 \mathrm{E}-08$ & Atm & $3.95 \mathrm{E}-08$ & Atm & $3.95 \mathrm{E}-08$ \\
\hline Co & $2.42 \mathrm{E}-06$ & $3.55 \mathrm{E}-09$ & $2.32 \mathrm{E}-05$ & $3.55 \mathrm{E}-09$ & $2.32 \mathrm{E}-05$ & $3.55 \mathrm{E}-09$ & $2.32 \mathrm{E}-05$ & $3.55 \mathrm{E}-09$ \\
\hline $\mathrm{Cu}$ & $3.87 \mathrm{E}-09$ & $1.05 \mathrm{E}-14$ & $3.90 \mathrm{E}-08$ & $1.05 \mathrm{E}-13$ & $3.90 \mathrm{E}-07$ & $1.05 \mathrm{E}-12$ & $3.90 \mathrm{E}-06$ & $1.05 \mathrm{E}-11$ \\
\hline $\mathrm{Fe}$ & $2.73 \mathrm{E}-07$ & $2.25 \mathrm{E}-18$ & $2.64 \mathrm{E}-06$ & $2.25 \mathrm{E}-18$ & $2.64 \mathrm{E}-06$ & $2.25 \mathrm{E}-18$ & $2.64 \mathrm{E}-06$ & $2.25 \mathrm{E}-18$ \\
\hline K & $4.72 \mathrm{E}-03$ & $4.68 \mathrm{E}-03$ & 4.72E-03 & $4.68 \mathrm{E}-03$ & $4.72 \mathrm{E}-03$ & $4.68 \mathrm{E}-03$ & $4.72 \mathrm{E}-03$ & $4.68 \mathrm{E}-03$ \\
\hline $\mathrm{Mg}$ & 8.39E-05 & $8.02 \mathrm{E}-05$ & 8.39E-05 & $8.02 E-05$ & 8.39E-05 & $8.02 \mathrm{E}-05$ & 8.39E-05 & $8.02 \mathrm{E}-05$ \\
\hline $\mathrm{Mn}$ & 8.60E-09 & $5.25 \mathrm{E}-09$ & $3.28 \mathrm{E}-08$ & $5.25 \mathrm{E}-09$ & $3.28 \mathrm{E}-08$ & $5.25 \mathrm{E}-09$ & $3.28 \mathrm{E}-08$ & $5.25 \mathrm{E}-09$ \\
\hline $\mathrm{MoO}_{4}$ & $3.00 \mathrm{E}-08$ & 2.99E-08 & $3.00 \mathrm{E}-08$ & $2.99 \mathrm{E}-08$ & $3.00 \mathrm{E}-08$ & 2.99E-08 & 3.00E-08 & $2.99 \mathrm{E}-08$ \\
\hline $\mathrm{Na}$ & 1.67E-02 & $1.66 \mathrm{E}-02$ & $1.67 \mathrm{E}-02$ & $1.66 \mathrm{E}-02$ & 1.67E-02 & $1.66 \mathrm{E}-02$ & 1.67E-02 & $1.66 \mathrm{E}-02$ \\
\hline $\mathrm{NH}_{4}$ & $9.37 \mathrm{E}-04$ & $9.32 \mathrm{E}-04$ & $9.37 \mathrm{E}-04$ & $9.32 \mathrm{E}-04$ & $9.37 \mathrm{E}-04$ & $9.32 \mathrm{E}-04$ & $9.37 \mathrm{E}-04$ & $9.32 \mathrm{E}-04$ \\
\hline $\mathrm{NO}_{3}$ & 1.60E-02 & $1.59 \mathrm{E}-02$ & $1.60 \mathrm{E}-02$ & $1.59 \mathrm{E}-02$ & 1.60E-02 & 1.59E-02 & 1.60E-02 & $1.59 \mathrm{E}-02$ \\
\hline $\mathrm{PO}_{4}$ & $1.37 \mathrm{E}-04$ & $5.25 \mathrm{E}-10$ & $1.37 \mathrm{E}-04$ & $5.25 \mathrm{E}-10$ & $1.37 \mathrm{E}-04$ & $5.25 \mathrm{E}-10$ & 1.37E-04 & $5.25 \mathrm{E}-10$ \\
\hline $\mathrm{SO}_{4}$ & $8.12 \mathrm{E}-05$ & $7.49 E-05$ & $8.12 \mathrm{E}-05$ & $7.49 E-05$ & $8.12 \mathrm{E}-05$ & 7.49E-05 & $8.12 \mathrm{E}-05$ & 7.49E-05 \\
\hline $\mathrm{Zn}$ & $8.25 \mathrm{E}-05$ & $9.55 \mathrm{E}-08$ & $7.83 \mathrm{E}-04$ & $9.55 \mathrm{E}-08$ & $7.83 \mathrm{E}-04$ & $9.55 \mathrm{E}-08$ & $7.85 \mathrm{E}-04$ & $9.55 \mathrm{E}-08$ \\
\hline NTA & $1.00-04$ & $8.44 \mathrm{E}-09$ & $1.00-04$ & $8.44 \mathrm{E}-09$ & $1.00-04$ & $8.44 \mathrm{E}-09$ & $1.05-04$ & 8.44E-09 \\
\hline MOPS & $1.00 \mathrm{E}-02$ & $4.31 \mathrm{E}-03$ & $1.00 \mathrm{E}-02$ & 4.31E-03 & $1.00 \mathrm{E}-02$ & 4.31E-03 & $1.00 \mathrm{E}-02$ & 4.31E-03 \\
\hline
\end{tabular}


Table S5: Ionic composition of LCo1 to LCo4 media. Free ion concentrations were calculated using MINEQL+ 5.0. All concentrations are given in molar (M).

\begin{tabular}{|c|c|c|c|c|c|c|c|c|}
\hline \multirow{2}{*}{$\begin{array}{l}\text { Media } \\
\text { Compound }\end{array}$} & \multicolumn{2}{|c|}{ LCo1 } & \multicolumn{2}{|c|}{ LCo2 } & \multicolumn{2}{|c|}{ LCo3 } & \multicolumn{2}{|c|}{ LCo 4} \\
\hline & Total & Free & Total & Free & Total & Free & Total & Free \\
\hline $\mathrm{BO}_{3}$ & $3.01 \mathrm{E}-06$ & $2.99 \mathrm{E}-06$ & $3.01 \mathrm{E}-06$ & $2.99 \mathrm{E}-06$ & $3.01 \mathrm{E}-06$ & $2.99 \mathrm{E}-06$ & $3.01 \mathrm{E}-06$ & $2.99 \mathrm{E}-06$ \\
\hline $\mathrm{Ca}$ & $1.06 \mathrm{E}-04$ & $9.60 \mathrm{E}-05$ & $1.06 \mathrm{E}-04$ & $9.60 \mathrm{E}-05$ & $1.06 \mathrm{E}-04$ & $9.60 \mathrm{E}-05$ & $1.06 \mathrm{E}-04$ & $9.60 \mathrm{E}-05$ \\
\hline $\mathrm{Cl}$ & $5.00 \mathrm{E}-06$ & 4.97E-06 & $5.00 \mathrm{E}-06$ & 4.97E-06 & $5.00 \mathrm{E}-06$ & 4.97E-06 & $5.00 \mathrm{E}-06$ & 4.97E-06 \\
\hline $\mathrm{CO}_{3}$ & Atm & $3.95 \mathrm{E}-08$ & Atm & $3.95 \mathrm{E}-08$ & Atm & $3.95 \mathrm{E}-08$ & Atm & $3.95 \mathrm{E}-08$ \\
\hline Co & $7.24 \mathrm{E}-09$ & $1.05 \mathrm{E}-11$ & $7.24 \mathrm{E}-09$ & $1.05 \mathrm{E}-11$ & $7.24 \mathrm{E}-09$ & $1.05 \mathrm{E}-11$ & $7.24 \mathrm{E}-09$ & $1.05 \mathrm{E}-11$ \\
\hline $\mathrm{Cu}$ & $3.92 \mathrm{E}-09$ & $1.05 \mathrm{E}-14$ & $3.92 \mathrm{E}-08$ & $1.05 \mathrm{E}-13$ & 3.92E-07 & $1.05 \mathrm{E}-12$ & $3.92 \mathrm{E}-06$ & $1.05 \mathrm{E}-11$ \\
\hline $\mathrm{Fe}$ & 2.77E-07 & $2.25 \mathrm{E}-18$ & 2.77E-07 & $2.25 \mathrm{E}-18$ & 2.77E-07 & $2.25 \mathrm{E}-18$ & 2.77E-07 & $2.25 \mathrm{E}-18$ \\
\hline $\mathrm{K}$ & $4.72 \mathrm{E}-03$ & $4.68 \mathrm{E}-03$ & $4.72 \mathrm{E}-03$ & $4.68 \mathrm{E}-03$ & $4.72 \mathrm{E}-03$ & $4.68 \mathrm{E}-03$ & $4.72 \mathrm{E}-03$ & $4.68 \mathrm{E}-03$ \\
\hline $\mathrm{Mg}$ & 8.39E-05 & 8.19E-05 & 8.39E-05 & 8.19E-05 & 8.39E-05 & 8.19E-05 & 8.39E-05 & $8.19 \mathrm{E}-05$ \\
\hline $\mathrm{Mn}$ & 3.07E-06 & $1.86 \mathrm{E}-06$ & $3.07 E-06$ & $1.86 \mathrm{E}-06$ & 3.07E-06 & $1.86 \mathrm{E}-06$ & 3.07E-06 & $1.86 \mathrm{E}-06$ \\
\hline $\mathrm{MoO}_{4}$ & $3.00 \mathrm{E}-08$ & 2.99E-08 & $3.00 \mathrm{E}-08$ & 2.99E-08 & 3.00E-08 & $2.99 \mathrm{E}-08$ & 3.00E-08 & $2.99 \mathrm{E}-08$ \\
\hline $\mathrm{Na}$ & 1.67E-02 & $1.66 \mathrm{E}-02$ & 1.67E-02 & $1.66 \mathrm{E}-02$ & 1.67E-02 & $1.66 \mathrm{E}-02$ & 1.67E-02 & $1.66 \mathrm{E}-02$ \\
\hline $\mathrm{NH}_{4}$ & 9.37E-04 & $9.32 \mathrm{E}-04$ & $9.37 E-04$ & $9.32 \mathrm{E}-04$ & $9.37 \mathrm{E}-04$ & $9.32 \mathrm{E}-04$ & $9.37 \mathrm{E}-04$ & $9.32 \mathrm{E}-04$ \\
\hline $\mathrm{NO}_{3}$ & $1.60 \mathrm{E}-02$ & $1.59 \mathrm{E}-02$ & $1.60 \mathrm{E}-02$ & $1.59 \mathrm{E}-02$ & $1.60 \mathrm{E}-02$ & $1.59 \mathrm{E}-02$ & $1.60 \mathrm{E}-02$ & $1.59 \mathrm{E}-02$ \\
\hline $\mathrm{PO}_{4}$ & $1.37 \mathrm{E}-04$ & $5.25 \mathrm{E}-10$ & $1.37 \mathrm{E}-04$ & $5.25 \mathrm{E}-10$ & 1.37E-04 & $5.25 \mathrm{E}-10$ & 1.37E-04 & $5.25 \mathrm{E}-10$ \\
\hline $\mathrm{SO}_{4}$ & $8.12 \mathrm{E}-05$ & $7.49 E-05$ & $8.12 \mathrm{E}-05$ & $7.49 \mathrm{E}-05$ & $8.12 \mathrm{E}-05$ & 7.49E-05 & $8.12 \mathrm{E}-05$ & 7.49E-05 \\
\hline $\mathrm{Zn}$ & $8.37 \mathrm{E}-05$ & $9.55 \mathrm{E}-08$ & 8.37E-05 & $9.55 \mathrm{E}-08$ & 8.37E-05 & $9.55 \mathrm{E}-08$ & 8.37E-05 & $9.55 \mathrm{E}-08$ \\
\hline NTA & $1.00 \mathrm{E}-04$ & 8.57E-09 & $1.00 \mathrm{E}-04$ & 8.57E-09 & $1.00 \mathrm{E}-04$ & 8.57E-09 & $1.03 \mathrm{E}-04$ & 8.57E-09 \\
\hline MOPS & $1.00 \mathrm{E}-02$ & $4.31 \mathrm{E}-03$ & $1.00 \mathrm{E}-02$ & $4.31 \mathrm{E}-03$ & $1.00 \mathrm{E}-02$ & $4.31 \mathrm{E}-03$ & $1.00 \mathrm{E}-02$ & $4.31 \mathrm{E}-03$ \\
\hline
\end{tabular}


Table S6: Ionic composition of LFe1 to LFe4 media. Free ion concentrations were calculated using MINEQL+5.0. All concentrations are given in molar $(\mathbf{M})$.

\begin{tabular}{|c|c|c|c|c|c|c|c|c|}
\hline \multirow{2}{*}{$\begin{array}{l}\text { Media } \\
\text { Compound }\end{array}$} & \multicolumn{2}{|c|}{ LFe1 } & \multicolumn{2}{|c|}{ LFe2 } & \multicolumn{2}{|c|}{ LFe3 } & \multicolumn{2}{|c|}{ LFe4 } \\
\hline & Total & Free & Total & Free & Total & Free & Total & Free \\
\hline $\mathrm{BO}_{3}$ & $3.01 \mathrm{E}-06$ & $2.99 \mathrm{E}-06$ & $3.01 \mathrm{E}-06$ & $2.99 \mathrm{E}-06$ & $3.01 \mathrm{E}-06$ & $2.99 \mathrm{E}-06$ & $3.01 \mathrm{E}-06$ & $2.99 \mathrm{E}-06$ \\
\hline $\mathrm{Ca}$ & $1.06 \mathrm{E}-04$ & $9.61 \mathrm{E}-05$ & $1.06 \mathrm{E}-04$ & $9.61 \mathrm{E}-05$ & $1.06 \mathrm{E}-04$ & $9.61 \mathrm{E}-05$ & $1.06 \mathrm{E}-04$ & $9.61 \mathrm{E}-05$ \\
\hline $\mathrm{Cl}$ & $5.00 \mathrm{E}-06$ & 4.97E-06 & $5.00 \mathrm{E}-06$ & 4.97E-06 & $5.00 \mathrm{E}-06$ & 4.97E-06 & $5.00 \mathrm{E}-06$ & 4.97E-06 \\
\hline $\mathrm{CO}_{3}$ & Atm & $3.95 \mathrm{E}-08$ & Atm & $3.95 \mathrm{E}-08$ & Atm & $3.95 \mathrm{E}-08$ & Atm & $3.95 \mathrm{E}-08$ \\
\hline Co & $2.46 \mathrm{E}-06$ & $3.55 E-9$ & $2.46 \mathrm{E}-06$ & $3.55 E-9$ & $2.46 \mathrm{E}-06$ & $3.55 E-9$ & $2.46 \mathrm{E}-06$ & 3.55E-9 \\
\hline $\mathrm{Cu}$ & $3.84 \mathrm{E}-09$ & $1.05 \mathrm{E}-14$ & $3.84 \mathrm{E}-08$ & $1.05 \mathrm{E}-13$ & $3.84 \mathrm{E}-07$ & $1.05 \mathrm{E}-12$ & $3.84 \mathrm{E}-06$ & $1.05 \mathrm{E}-11$ \\
\hline $\mathrm{Fe}$ & $1.33 \mathrm{E}-08$ & $1.10 \mathrm{E}-19$ & $1.33 \mathrm{E}-08$ & $1.10 \mathrm{E}-19$ & 1.33E-08 & 1.10E-19 & $1.33 \mathrm{E}-08$ & $1.10 \mathrm{E}-19$ \\
\hline K & $4.72 \mathrm{E}-03$ & $4.68 \mathrm{E}-03$ & $4.72 \mathrm{E}-03$ & $4.68 \mathrm{E}-03$ & $4.72 \mathrm{E}-03$ & 4.68E-03 & $4.72 \mathrm{E}-03$ & $4.68 \mathrm{E}-03$ \\
\hline $\mathrm{Mg}$ & $8.39 \mathrm{E}-05$ & $8.19 E-05$ & 8.39E-05 & 8.19E-05 & 8.39E-05 & 8.19E-05 & $8.39 \mathrm{E}-05$ & $8.19 \mathrm{E}-05$ \\
\hline $\mathrm{Mn}$ & $3.04 \mathrm{E}-06$ & $1.86 \mathrm{E}-06$ & $3.04 \mathrm{E}-06$ & $1.86 \mathrm{E}-06$ & $3.04 \mathrm{E}-06$ & $1.86 \mathrm{E}-06$ & $3.04 \mathrm{E}-06$ & $1.86 \mathrm{E}-06$ \\
\hline $\mathrm{MoO}_{4}$ & $3.00 \mathrm{E}-08$ & $2.99 \mathrm{E}-08$ & $3.00 \mathrm{E}-08$ & 2.99E-08 & $3.00 \mathrm{E}-08$ & $2.99 \mathrm{E}-08$ & $3.00 \mathrm{E}-08$ & $2.99 \mathrm{E}-08$ \\
\hline $\mathrm{Na}$ & 1.67E-02 & $1.66 \mathrm{E}-02$ & 1.67E-02 & $1.66 \mathrm{E}-02$ & 1.67E-02 & $1.66 \mathrm{E}-02$ & 1.67E-02 & $1.66 \mathrm{E}-02$ \\
\hline $\mathrm{NH}_{4}$ & $9.37 \mathrm{E}-04$ & $9.32 \mathrm{E}-04$ & $9.37 \mathrm{E}-04$ & $9.32 \mathrm{E}-04$ & $9.37 \mathrm{E}-04$ & $9.32 \mathrm{E}-04$ & 9.37E-04 & $9.32 \mathrm{E}-04$ \\
\hline $\mathrm{NO}_{3}$ & $1.60 \mathrm{E}-02$ & $1.59 \mathrm{E}-02$ & $1.60 \mathrm{E}-02$ & $1.59 \mathrm{E}-02$ & $1.60 \mathrm{E}-02$ & $1.59 \mathrm{E}-02$ & $1.60 \mathrm{E}-02$ & $1.59 \mathrm{E}-02$ \\
\hline $\mathrm{PO}_{4}$ & $1.37 \mathrm{E}-04$ & $5.25 \mathrm{E}-10$ & $1.37 \mathrm{E}-04$ & $5.25 \mathrm{E}-10$ & 1.37E-04 & $5.25 \mathrm{E}-10$ & $1.37 \mathrm{E}-04$ & $5.25 \mathrm{E}-10$ \\
\hline $\mathrm{SO}_{4}$ & $8.12 \mathrm{E}-05$ & $7.49 \mathrm{E}-05$ & $8.12 \mathrm{E}-05$ & 7.49E-05 & $8.12 \mathrm{E}-05$ & 7.49E-05 & $8.12 \mathrm{E}-05$ & $7.49 \mathrm{E}-05$ \\
\hline $\mathrm{Zn}$ & 8.19E-05 & $9.55 \mathrm{E}-08$ & $8.19 \mathrm{E}-05$ & $9.55 \mathrm{E}-08$ & 8.19E-05 & $9.55 \mathrm{E}-08$ & 8.19E-05 & $9.55 \mathrm{E}-08$ \\
\hline NTA & $1.00 \mathrm{E}-04$ & 8.37E-09 & $1.00 \mathrm{E}-04$ & 8.37E-09 & 1.00E-04 & 8.37E-09 & $1.03 \mathrm{E}-04$ & 8.37E-09 \\
\hline MOPS & $1.00 \mathrm{E}-02$ & $4.31 \mathrm{E}-03$ & $1.00 \mathrm{E}-02$ & 4.31E-03 & $1.00 \mathrm{E}-02$ & 4.31E-03 & $1.00 \mathrm{E}-02$ & $4.31 \mathrm{E}-03$ \\
\hline
\end{tabular}


Table S7: Ionic composition of HM1 to HM10 media. Free ion concentrations were calculated using MINEQL+ 5.0. All concentrations are given in molar (M).

\begin{tabular}{|c|c|c|c|c|c|c|c|c|c|c|}
\hline \multirow{2}{*}{$\begin{array}{c}\text { Media } \\
\text { Compound }\end{array}$} & \multicolumn{2}{|c|}{ HM1 } & \multicolumn{2}{|c|}{ HM2 } & \multicolumn{2}{|c|}{ HM3 } & \multicolumn{2}{|c|}{ HM4 } & \multicolumn{2}{|c|}{ HM5 } \\
\hline & Total & Free & Total & Free & Total & Free & Total & Free & Total & Free \\
\hline $\mathrm{BO}_{3}$ & $3.01 \mathrm{E}-06$ & $2.72 \mathrm{E}-06$ & 3.01E-06 & $2.72 \mathrm{E}-06$ & $3.01 \mathrm{E}-06$ & $2.72 \mathrm{E}-06$ & 3.01E-06 & $2.72 \mathrm{E}-06$ & $3.01 \mathrm{E}-06$ & $2.72 \mathrm{E}-06$ \\
\hline $\mathrm{Ca}$ & $7.36 \mathrm{E}-05$ & $6.57 \mathrm{E}-05$ & 7.36E-05 & $6.57 \mathrm{E}-05$ & 7.36E-05 & 6.57E-05 & 7.36E-05 & $6.57 \mathrm{E}-05$ & 7.36E-05 & $6.57 \mathrm{E}-05$ \\
\hline $\mathrm{Cl}$ & 5.98E-06 & 5.95E-06 & 5.98E-06 & 5.95E-06 & 5.98E-06 & 5.95E-06 & 5.98E-06 & 5.95E-06 & 5.98E-06 & 5.95E-06 \\
\hline $\mathrm{CO}_{3}$ & Atm & $3.68 \mathrm{E}-08$ & Atm & $3.68 \mathrm{E}-08$ & Atm & $3.68 \mathrm{E}-08$ & Atm & $3.68 \mathrm{E}-08$ & Atm & $3.68 \mathrm{E}-08$ \\
\hline Co & $2.53 \mathrm{E}-06$ & $3.55 \mathrm{E}-09$ & $2.53 \mathrm{E}-06$ & $3.55 \mathrm{E}-09$ & $2.53 \mathrm{E}-06$ & $3.55 \mathrm{E}-09$ & $2.53 \mathrm{E}-06$ & $3.55 \mathrm{E}-09$ & $2.53 \mathrm{E}-06$ & $3.55 \mathrm{E}-09$ \\
\hline $\mathrm{Cu}$ & $4.25 \mathrm{E}-09$ & $1.05 \mathrm{E}-14$ & $4.25 \mathrm{E}-08$ & $1.05 \mathrm{E}-13$ & $4.25 \mathrm{E}-07$ & $1.05 \mathrm{E}-12$ & $4.25 \mathrm{E}-06$ & $1.05 \mathrm{E}-11$ & $4.25 \mathrm{E}-05$ & $1.05 \mathrm{E}-10$ \\
\hline $\mathrm{Fe}$ & $5.70 \mathrm{E}-06$ & $2.25 \mathrm{E}-18$ & $5.70 \mathrm{E}-06$ & $2.25 \mathrm{E}-18$ & $5.70 \mathrm{E}-06$ & $2.25 \mathrm{E}-18$ & $5.70 \mathrm{E}-06$ & $2.25 \mathrm{E}-18$ & $5.70 \mathrm{E}-06$ & $2.25 \mathrm{E}-18$ \\
\hline K & $4.22 \mathrm{E}-03$ & $4.21 \mathrm{E}-03$ & $4.22 \mathrm{E}-03$ & $4.21 \mathrm{E}-03$ & $4.22 \mathrm{E}-03$ & $4.21 \mathrm{E}-03$ & $4.22 \mathrm{E}-03$ & $4.21 \mathrm{E}-03$ & $4.22 \mathrm{E}-03$ & $4.21 \mathrm{E}-03$ \\
\hline $\mathrm{Mg}$ & 8.07E-05 & 7.69E-05 & 8.07E-05 & 7.69E-05 & 8.07E-05 & 7.69E-05 & 8.07E-05 & 7.69E-05 & 8.07E-05 & $7.69 \mathrm{E}-05$ \\
\hline $\mathrm{Mn}$ & $3.21 \mathrm{E}-06$ & $1.86 \mathrm{E}-06$ & $3.21 \mathrm{E}-06$ & $1.86 \mathrm{E}-06$ & $3.21 \mathrm{E}-06$ & $1.86 \mathrm{E}-06$ & $3.21 \mathrm{E}-06$ & $1.86 \mathrm{E}-06$ & $3.21 \mathrm{E}-06$ & $1.86 \mathrm{E}-06$ \\
\hline $\mathrm{MoO}_{4}$ & $3.00 \mathrm{E}-08$ & $3.00 \mathrm{E}-08$ & $3.00 \mathrm{E}-08$ & $3.00 \mathrm{E}-08$ & $3.00 \mathrm{E}-08$ & $3.00 \mathrm{E}-08$ & $3.00 \mathrm{E}-08$ & $3.00 \mathrm{E}-08$ & $3.00 \mathrm{E}-08$ & $3.00 \mathrm{E}-08$ \\
\hline $\mathrm{Na}$ & $1.67 \mathrm{E}-02$ & $1.67 \mathrm{E}-02$ & 1.67E-02 & 1.67E-02 & 1.67E-02 & 1.67E-02 & 1.67E-02 & 1.67E-02 & 1.67E-02 & 1.67E-02 \\
\hline $\mathrm{NH}_{4}$ & $9.37 \mathrm{E}-04$ & $8.70 \mathrm{E}-04$ & $9.37 \mathrm{E}-04$ & $8.70 \mathrm{E}-04$ & $9.37 \mathrm{E}-04$ & $8.70 \mathrm{E}-04$ & $9.37 \mathrm{E}-04$ & 8.70E-04 & 9.37E-04 & 8.70E-04 \\
\hline $\mathrm{NO}_{3}$ & 5.07E-03 & $5.04 \mathrm{E}-03$ & 5.07E-03 & $5.04 \mathrm{E}-03$ & 5.07E-03 & $5.04 \mathrm{E}-03$ & 5.07E-03 & $5.04 \mathrm{E}-03$ & 5.07E-03 & $5.04 \mathrm{E}-03$ \\
\hline $\mathrm{PO}_{4}$ & $1.37 \mathrm{E}-04$ & $1.35 \mathrm{E}-08$ & $1.37 \mathrm{E}-04$ & $1.35 \mathrm{E}-08$ & 1.37E-04 & $1.35 \mathrm{E}-08$ & $1.37 \mathrm{E}-04$ & $1.35 \mathrm{E}-08$ & $1.37 \mathrm{E}-04$ & $1.35 \mathrm{E}-08$ \\
\hline $\mathrm{SO}_{4}$ & $8.12 \mathrm{E}-05$ & $7.49 \mathrm{E}-05$ & $8.12 \mathrm{E}-05$ & 7.49E-05 & $8.12 \mathrm{E}-05$ & $7.49 \mathrm{E}-05$ & $8.12 \mathrm{E}-05$ & $7.49 \mathrm{E}-05$ & $8.12 \mathrm{E}-05$ & $7.49 \mathrm{E}-05$ \\
\hline $\mathrm{Zn}$ & $8.70 \mathrm{E}-05$ & $9.56 \mathrm{E}-08$ & $8.70 \mathrm{E}-05$ & $9.56 \mathrm{E}-08$ & $8.70 \mathrm{E}-05$ & $9.56 \mathrm{E}-08$ & $8.70 \mathrm{E}-05$ & $9.56 \mathrm{E}-08$ & $8.70 \mathrm{E}-05$ & $9.56 \mathrm{E}-08$ \\
\hline NTA & $1.00 \mathrm{E}-04$ & 7.98E-09 & $1.00 \mathrm{E}-04$ & 7.98E-09 & $1.00 \mathrm{E}-04$ & 7.98E-09 & $1.04 \mathrm{E}-04$ & 7.98E-09 & $1.42 \mathrm{E}-04$ & 7.98E-09 \\
\hline MOPS & $1.00 \mathrm{E}-02$ & $9.21 \mathrm{E}-03$ & $1.00 \mathrm{E}-02$ & $9.21 \mathrm{E}-03$ & $1.00 \mathrm{E}-02$ & $9.21 \mathrm{E}-03$ & $1.00 \mathrm{E}-02$ & $9.21 \mathrm{E}-03$ & $1.00 \mathrm{E}-02$ & $9.21 \mathrm{E}-03$ \\
\hline
\end{tabular}


Table S7: Ionic composition of HM1 to HM10 media. Free ion concentrations were calculated using MINEQL+ 5.0. All concentrations are given in molar (M). (continued)

\begin{tabular}{|c|c|c|c|c|c|c|c|c|c|c|}
\hline Media & & 16 & & 17 & & 18 & & M9 & & 110 \\
\hline Compound & Total & Free & Total & Free & Total & Free & Total & Free & Total & Free \\
\hline $\mathrm{BO}_{3}$ & $3.01 \mathrm{E}-06$ & $2.72 \mathrm{E}-06$ & 3.01E-06 & $2.72 \mathrm{E}-06$ & $3.01 \mathrm{E}-06$ & $2.72 \mathrm{E}-06$ & $3.01 \mathrm{E}-06$ & $2.72 \mathrm{E}-06$ & $3.01 \mathrm{E}-06$ & $2.72 \mathrm{E}-06$ \\
\hline $\mathrm{Ca}$ & $7.36 \mathrm{E}-05$ & $6.57 \mathrm{E}-05$ & 7.36E-05 & $6.57 \mathrm{E}-05$ & 7.36E-05 & 6.57E-05 & 7.36E-05 & $6.57 \mathrm{E}-05$ & 7.36E-05 & $.57 E-05$ \\
\hline $\mathrm{Cl}$ & 5.98E-06 & 5.95E-06 & 5.98E-06 & 5.95E-06 & 5.98E-06 & 5.95E-06 & 5.98E-06 & 5.95E-06 & 5.98E-06 & 5.95E-06 \\
\hline $\mathrm{CO}_{3}$ & Atm & $3.68 \mathrm{E}-08$ & Atm & $3.68 \mathrm{E}-08$ & Atm & $3.68 \mathrm{E}-08$ & Atm & $3.68 \mathrm{E}-08$ & Atm & $3.68 \mathrm{E}-08$ \\
\hline Co & $2.53 \mathrm{E}-06$ & $3.55 \mathrm{E}-09$ & $2.53 \mathrm{E}-06$ & $3.55 \mathrm{E}-09$ & $2.53 \mathrm{E}-06$ & $3.55 \mathrm{E}-09$ & $2.53 \mathrm{E}-06$ & $3.55 \mathrm{E}-09$ & $2.53 \mathrm{E}-06$ & $3.55 \mathrm{E}-09$ \\
\hline $\mathrm{Cu}$ & $4.25 \mathrm{E}-04$ & $1.05 \mathrm{E}-09$ & $6.04 \mathrm{E}-04$ & $1.51 \mathrm{E}-09$ & $9.94 \mathrm{E}-04$ & $2.51 \mathrm{E}-09$ & $1.36 \mathrm{E}-03$ & 3.47E-09 & $3.91 \mathrm{E}-03$ & $1.05 \mathrm{E}-08$ \\
\hline $\mathrm{Fe}$ & $5.70 \mathrm{E}-06$ & $2.25 \mathrm{E}-18$ & $5.70 \mathrm{E}-06$ & $2.25 \mathrm{E}-18$ & $5.70 \mathrm{E}-06$ & $2.25 \mathrm{E}-18$ & $5.70 \mathrm{E}-06$ & & $5.70 \mathrm{E}-06$ & $2.25 \mathrm{E}-18$ \\
\hline K & $4.22 \mathrm{E}-03$ & $4.21 \mathrm{E}-03$ & $4.22 \mathrm{E}-03$ & $4.21 \mathrm{E}-03$ & $4.22 \mathrm{E}-03$ & $4.21 \mathrm{E}-03$ & $4.22 \mathrm{E}-03$ & $4.21 \mathrm{E}-03$ & $4.22 \mathrm{E}-03$ & $4.21 \mathrm{E}-03$ \\
\hline $\mathrm{Mg}$ & 8.07E-05 & 7.69E-05 & 8.07E-05 & 7.69E-05 & 8.07E-05 & 7.69E-05 & 8.07E-05 & 7.69E-05 & 8.07E-05 & $7.69 \mathrm{E}-05$ \\
\hline $\mathrm{Mn}$ & $3.21 \mathrm{E}-06$ & $1.86 \mathrm{E}-06$ & $3.21 \mathrm{E}-06$ & $1.86 \mathrm{E}-06$ & $3.21 \mathrm{E}-06$ & $1.86 \mathrm{E}-06$ & $3.21 \mathrm{E}-06$ & $1.86 \mathrm{E}-06$ & $3.21 \mathrm{E}-06$ & $1.86 \mathrm{E}-06$ \\
\hline $\mathrm{MoO}_{4}$ & $3.00 \mathrm{E}-08$ & $3.00 \mathrm{E}-08$ & $3.00 \mathrm{E}-08$ & $3.00 \mathrm{E}-08$ & $3.00 \mathrm{E}-08$ & $3.00 \mathrm{E}-08$ & $3.00 \mathrm{E}-08$ & $3.00 \mathrm{E}-08$ & $3.00 \mathrm{E}-08$ & $3.00 \mathrm{E}-08$ \\
\hline $\mathrm{Na}$ & 1.67E-02 & $1.67 \mathrm{E}-02$ & 1.67E-02 & 1.67E-02 & 1.67E-02 & 1.67E-02 & 1.67E-02 & 1.67E-02 & 1.67E-02 & 1.67E-02 \\
\hline $\mathrm{NH}_{4}$ & $9.37 \mathrm{E}-04$ & $8.70 \mathrm{E}-04$ & $9.37 \mathrm{E}-04$ & $8.70 \mathrm{E}-04$ & $9.37 \mathrm{E}-04$ & $8.70 \mathrm{E}-04$ & $9.37 \mathrm{E}-04$ & 8.70E-04 & 9.37E-04 & 8.70E-04 \\
\hline $\mathrm{NO}_{3}$ & 5.07E-03 & $5.04 \mathrm{E}-03$ & 5.07E-03 & $5.04 \mathrm{E}-03$ & 5.07E-03 & $5.04 \mathrm{E}-03$ & 5.07E-03 & $5.04 \mathrm{E}-03$ & 5.07E-03 & $5.04 \mathrm{E}-03$ \\
\hline $\mathrm{PO}_{4}$ & $1.37 \mathrm{E}-04$ & $1.35 \mathrm{E}-08$ & $1.37 \mathrm{E}-04$ & $1.35 \mathrm{E}-08$ & 1.37E-04 & $1.35 \mathrm{E}-08$ & $1.37 \mathrm{E}-04$ & $1.35 \mathrm{E}-08$ & $1.37 \mathrm{E}-04$ & $1.35 \mathrm{E}-08$ \\
\hline $\mathrm{SO}_{4}$ & $8.12 \mathrm{E}-05$ & $7.49 \mathrm{E}-05$ & $8.12 \mathrm{E}-05$ & 7.49E-05 & $8.12 \mathrm{E}-05$ & $7.49 \mathrm{E}-05$ & $8.12 \mathrm{E}-05$ & $7.49 \mathrm{E}-05$ & $8.12 \mathrm{E}-05$ & $7.49 \mathrm{E}-05$ \\
\hline $\mathrm{Zn}$ & $8.70 \mathrm{E}-05$ & $9.56 \mathrm{E}-08$ & $8.70 \mathrm{E}-05$ & $9.56 \mathrm{E}-08$ & $8.70 \mathrm{E}-05$ & $9.56 \mathrm{E}-08$ & $8.70 \mathrm{E}-05$ & $9.56 \mathrm{E}-08$ & $8.70 \mathrm{E}-05$ & $9.56 \mathrm{E}-08$ \\
\hline NTA & $5.18 \mathrm{E}-04$ & 7.98E-09 & $7.02 \mathrm{E}-04$ & 7.98E-09 & $1.09 \mathrm{E}-03$ & 7.98E-09 & $1.46 \mathrm{E}-03$ & 7.98E-09 & $4.00 \mathrm{E}-03$ & 7.98E-09 \\
\hline MOPS & $1.00 \mathrm{E}-02$ & $9.21 \mathrm{E}-03$ & $1.00 \mathrm{E}-02$ & $9.21 \mathrm{E}-03$ & $1.00 \mathrm{E}-02$ & $9.21 \mathrm{E}-03$ & $1.00 \mathrm{E}-02$ & $9.21 \mathrm{E}-03$ & $1.00 \mathrm{E}-02$ & $9.21 \mathrm{E}-03$ \\
\hline
\end{tabular}


Table S8: Ionic composition of LFe1 to LFe10 media. Free ion concentrations were calculated using MINEQL+ 5.0. All concentrations are given in molar (M).

\begin{tabular}{|c|c|c|c|c|c|c|c|c|c|c|}
\hline \multirow{2}{*}{$\begin{array}{l}\text { Media } \\
\text { Compound }\end{array}$} & \multicolumn{2}{|c|}{ LFe1 } & \multicolumn{2}{|c|}{ LFe2 } & \multicolumn{2}{|c|}{ LFe3 } & \multicolumn{2}{|c|}{ LFe4 } & \multicolumn{2}{|c|}{ LFe5 } \\
\hline & Total & Free & Total & Free & Total & Free & Total & Free & Total & Free \\
\hline $\mathrm{BO}_{3}$ & $3.01 \mathrm{E}-06$ & $2.99 \mathrm{E}-06$ & $3.01 \mathrm{E}-06$ & $2.99 \mathrm{E}-06$ & $3.01 \mathrm{E}-06$ & $2.99 \mathrm{E}-06$ & $3.01 \mathrm{E}-06$ & $2.99 \mathrm{E}-06$ & $3.01 \mathrm{E}-06$ & $2.99 \mathrm{E}-06$ \\
\hline $\mathrm{Ca}$ & $1.06 \mathrm{E}-04$ & 9.61E-05 & $1.06 \mathrm{E}-04$ & 9.61E-05 & $1.06 \mathrm{E}-04$ & $9.61 \mathrm{E}-05$ & $1.06 \mathrm{E}-04$ & 9.61E-05 & $1.06 \mathrm{E}-04$ & $9.61 \mathrm{E}-05$ \\
\hline $\mathrm{Cl}$ & $5.00 \mathrm{E}-06$ & 4.97E-06 & $5.00 \mathrm{E}-06$ & 4.97E-06 & $5.00 \mathrm{E}-06$ & 4.97E-06 & $5.00 \mathrm{E}-06$ & 4.97E-06 & $5.00 \mathrm{E}-06$ & 4.97E-06 \\
\hline $\mathrm{CO}_{3}$ & Atm & $3.95 \mathrm{E}-08$ & Atm & $3.95 \mathrm{E}-08$ & Atm & $3.95 \mathrm{E}-08$ & Atm & $3.95 \mathrm{E}-08$ & Atm & $3.95 \mathrm{E}-08$ \\
\hline Co & $2.46 \mathrm{E}-06$ & $3.55 E-9$ & $2.46 \mathrm{E}-06$ & $3.55 E-9$ & $2.46 \mathrm{E}-06$ & $3.55 E-9$ & $2.46 \mathrm{E}-06$ & $3.55 E-9$ & $2.46 \mathrm{E}-06$ & $3.55 E-9$ \\
\hline $\mathrm{Cu}$ & $3.84 \mathrm{E}-08$ & $1.05 \mathrm{E}-13$ & 1.10E-07 & $3.02 \mathrm{E}-13$ & 2.19E-07 & $6.03 \mathrm{E}-13$ & $2.89 \mathrm{E}-07$ & $7.94 \mathrm{E}-13$ & $3.84 \mathrm{E}-07$ & $1.05 \mathrm{E}-12$ \\
\hline $\mathrm{Fe}$ & $1.33 \mathrm{E}-08$ & 1.10E-19 & $1.33 \mathrm{E}-08$ & $1.10 \mathrm{E}-19$ & $1.33 \mathrm{E}-08$ & $1.10 \mathrm{E}-19$ & $1.33 \mathrm{E}-08$ & 1.10E-19 & $1.33 \mathrm{E}-08$ & $1.10 \mathrm{E}-19$ \\
\hline K & 4.72E-03 & 4.68E-03 & 4.72E-03 & $4.68 \mathrm{E}-03$ & 4.72E-03 & 4.68E-03 & 4.72E-03 & 4.68E-03 & 4.72E-03 & 4.68E-03 \\
\hline $\mathrm{Mg}$ & $8.39 \mathrm{E}-05$ & $8.19 \mathrm{E}-05$ & 8.39E-05 & $8.19 E-05$ & 8.39E-05 & $8.19 \mathrm{E}-05$ & $8.39 \mathrm{E}-05$ & $8.19 \mathrm{E}-05$ & 8.39E-05 & $8.19 \mathrm{E}-05$ \\
\hline $\mathrm{Mn}$ & $3.04 \mathrm{E}-06$ & $1.86 \mathrm{E}-06$ & $3.04 \mathrm{E}-06$ & $1.86 \mathrm{E}-06$ & $3.04 \mathrm{E}-06$ & $1.86 \mathrm{E}-06$ & $3.04 \mathrm{E}-06$ & $1.86 \mathrm{E}-06$ & $3.04 \mathrm{E}-06$ & $1.86 \mathrm{E}-06$ \\
\hline $\mathrm{MoO}_{4}$ & $3.00 \mathrm{E}-08$ & $2.99 \mathrm{E}-08$ & $3.00 \mathrm{E}-08$ & 2.99E-08 & $3.00 \mathrm{E}-08$ & 2.99E-08 & 3.00E-08 & $2.99 \mathrm{E}-08$ & $3.00 \mathrm{E}-08$ & $2.99 \mathrm{E}-08$ \\
\hline $\mathrm{Na}$ & 1.67E-02 & $1.66 \mathrm{E}-02$ & 1.67E-02 & $1.66 \mathrm{E}-02$ & 1.67E-02 & 1.66E-02 & 1.67E-02 & $1.66 \mathrm{E}-02$ & 1.67E-02 & $1.66 \mathrm{E}-02$ \\
\hline $\mathrm{NH}_{4}$ & $9.37 \mathrm{E}-04$ & $9.32 \mathrm{E}-04$ & $9.37 \mathrm{E}-04$ & $9.32 \mathrm{E}-04$ & $9.37 \mathrm{E}-04$ & $9.32 \mathrm{E}-04$ & $9.37 \mathrm{E}-04$ & $9.32 \mathrm{E}-04$ & $9.37 \mathrm{E}-04$ & $9.32 \mathrm{E}-04$ \\
\hline $\mathrm{NO}_{3}$ & $1.60 \mathrm{E}-02$ & $1.59 \mathrm{E}-02$ & $1.60 \mathrm{E}-02$ & $1.59 \mathrm{E}-02$ & $1.60 \mathrm{E}-02$ & $1.59 \mathrm{E}-02$ & $1.60 \mathrm{E}-02$ & $1.59 \mathrm{E}-02$ & $1.60 \mathrm{E}-02$ & $1.59 \mathrm{E}-02$ \\
\hline $\mathrm{PO}_{4}$ & $1.37 \mathrm{E}-04$ & $5.25 \mathrm{E}-10$ & $1.37 \mathrm{E}-04$ & $5.25 \mathrm{E}-10$ & $1.37 \mathrm{E}-04$ & $5.25 \mathrm{E}-10$ & $1.37 \mathrm{E}-04$ & $5.25 \mathrm{E}-10$ & $1.37 \mathrm{E}-04$ & $5.25 \mathrm{E}-10$ \\
\hline $\mathrm{SO}_{4}$ & $8.12 \mathrm{E}-05$ & $7.49 \mathrm{E}-05$ & $8.12 \mathrm{E}-05$ & $7.49 \mathrm{E}-05$ & $8.12 \mathrm{E}-05$ & $7.49 \mathrm{E}-05$ & $8.12 \mathrm{E}-05$ & $7.49 E-05$ & $8.12 \mathrm{E}-05$ & $7.49 \mathrm{E}-05$ \\
\hline $\mathrm{Zn}$ & 8.19E-05 & $9.55 \mathrm{E}-08$ & $8.19 \mathrm{E}-05$ & $9.55 \mathrm{E}-08$ & 8.19E-05 & $9.55 \mathrm{E}-08$ & 8.19E-05 & $9.55 \mathrm{E}-08$ & 8.19E-05 & $9.55 \mathrm{E}-08$ \\
\hline NTA & $1.00 \mathrm{E}-04$ & 8.37E-09 & $1.00 \mathrm{E}-04$ & 8.37E-09 & $1.00 \mathrm{E}-04$ & 8.37E-09 & $1.00 \mathrm{E}-04$ & 8.37E-09 & $1.00 \mathrm{E}-04$ & 8.37E-09 \\
\hline MOPS & $1.00 \mathrm{E}-02$ & 4.31E-03 & $1.00 \mathrm{E}-02$ & 4.31E-03 & $1.00 \mathrm{E}-02$ & 4.31E-03 & $1.00 \mathrm{E}-02$ & 4.31E-03 & $1.00 \mathrm{E}-02$ & 4.31E-03 \\
\hline
\end{tabular}


Table S8 (continued): Ionic composition of LFe1 to LFe10 media. Free ion concentrations were calculated using MINEQL+ 5.0. All concentrations are given in molar (M).

\begin{tabular}{|c|c|c|c|c|c|c|c|c|c|c|}
\hline \multirow{2}{*}{$\begin{array}{l}\text { Media } \\
\text { Compound }\end{array}$} & \multicolumn{2}{|c|}{ LFe6 } & \multicolumn{2}{|c|}{ LFe7 } & \multicolumn{2}{|c|}{ LFe8 } & \multicolumn{2}{|c|}{ LFe9 } & \multicolumn{2}{|c|}{ LFe10 } \\
\hline & Total & Free & Total & Free & Total & Free & Total & Free & Total & Free \\
\hline $\mathrm{BO}_{3}$ & $3.01 \mathrm{E}-06$ & 2.99E-06 & $3.01 \mathrm{E}-06$ & 2.99E-06 & $3.01 \mathrm{E}-06$ & $2.99 \mathrm{E}-06$ & $3.01 \mathrm{E}-06$ & 2.99E-06 & $3.01 \mathrm{E}-06$ & $2.99 \mathrm{E}-06$ \\
\hline $\mathrm{Ca}$ & $1.06 \mathrm{E}-04$ & $9.61 \mathrm{E}-05$ & $1.06 \mathrm{E}-04$ & $9.61 \mathrm{E}-05$ & $1.06 \mathrm{E}-04$ & 9.61E-05 & $1.06 \mathrm{E}-04$ & $9.61 \mathrm{E}-05$ & $1.06 \mathrm{E}-04$ & $9.61 E-05$ \\
\hline $\mathrm{Cl}$ & $5.00 \mathrm{E}-06$ & 4.97E-06 & $5.00 \mathrm{E}-06$ & 4.97E-06 & $5.00 \mathrm{E}-06$ & 4.97E-06 & $5.00 \mathrm{E}-06$ & 4.97E-06 & $5.00 \mathrm{E}-06$ & 4.97E-06 \\
\hline $\mathrm{CO}_{3}$ & Atm & $3.95 \mathrm{E}-08$ & Atm & $3.95 \mathrm{E}-08$ & Atm & $3.95 \mathrm{E}-08$ & Atm & $3.95 \mathrm{E}-08$ & Atm & $3.95 \mathrm{E}-08$ \\
\hline Co & $2.46 \mathrm{E}-06$ & $3.55 E-9$ & $2.46 \mathrm{E}-06$ & $3.55 E-9$ & $2.46 \mathrm{E}-06$ & $3.55 E-9$ & $2.46 \mathrm{E}-06$ & $3.55 E-9$ & $2.46 \mathrm{E}-06$ & $3.55 E-9$ \\
\hline $\mathrm{Cu}$ & $1.45 \mathrm{E}-06$ & $3.98 \mathrm{E}-12$ & $2.35 \mathrm{E}-06$ & $6.46 \mathrm{E}-12$ & $2.89 \mathrm{E}-06$ & $7.94 \mathrm{E}-12$ & $3.81 \mathrm{E}-06$ & $1.05 \mathrm{E}-11$ & $1.05 \mathrm{E}-05$ & $2.88 \mathrm{E}-11$ \\
\hline $\mathrm{Fe}$ & $1.33 \mathrm{E}-08$ & $1.10 \mathrm{E}-19$ & $1.33 \mathrm{E}-08$ & $1.10 \mathrm{E}-19$ & $1.33 \mathrm{E}-08$ & $1.10 \mathrm{E}-19$ & $1.33 \mathrm{E}-08$ & $1.10 \mathrm{E}-19$ & $1.33 \mathrm{E}-08$ & $1.10 \mathrm{E}-19$ \\
\hline K & $4.72 \mathrm{E}-03$ & $4.68 \mathrm{E}-03$ & $4.72 \mathrm{E}-03$ & $4.68 \mathrm{E}-03$ & $4.72 \mathrm{E}-03$ & $4.68 \mathrm{E}-03$ & $4.72 \mathrm{E}-03$ & $4.68 \mathrm{E}-03$ & $4.72 \mathrm{E}-03$ & $4.68 \mathrm{E}-03$ \\
\hline $\mathrm{Mg}$ & 8.39E-05 & 8.19E-05 & 8.39E-05 & 8.19E-05 & 8.39E-05 & $8.19 E-05$ & $8.39 \mathrm{E}-05$ & 8.19E-05 & $8.39 \mathrm{E}-05$ & $8.19 \mathrm{E}-05$ \\
\hline $\mathrm{Mn}$ & $3.04 \mathrm{E}-06$ & $1.86 \mathrm{E}-06$ & $3.04 \mathrm{E}-06$ & $1.86 \mathrm{E}-06$ & $3.04 \mathrm{E}-06$ & $1.86 \mathrm{E}-06$ & $3.04 \mathrm{E}-06$ & $1.86 \mathrm{E}-06$ & $3.04 \mathrm{E}-06$ & $1.86 \mathrm{E}-06$ \\
\hline $\mathrm{MoO}_{4}$ & $3.00 \mathrm{E}-08$ & 2.99E-08 & $3.00 \mathrm{E}-08$ & 2.99E-08 & $3.00 \mathrm{E}-08$ & 2.99E-08 & $3.00 \mathrm{E}-08$ & 2.99E-08 & $3.00 \mathrm{E}-08$ & $2.99 \mathrm{E}-08$ \\
\hline $\mathrm{Na}$ & 1.67E-02 & $1.66 \mathrm{E}-02$ & 1.67E-02 & $1.66 \mathrm{E}-02$ & $1.67 \mathrm{E}-02$ & $1.66 \mathrm{E}-02$ & 1.67E-02 & $1.66 \mathrm{E}-02$ & 1.67E-02 & $1.66 \mathrm{E}-02$ \\
\hline $\mathrm{NH}_{4}$ & 9.37E-04 & $9.32 \mathrm{E}-04$ & $9.37 \mathrm{E}-04$ & $9.32 \mathrm{E}-04$ & $9.37 \mathrm{E}-04$ & $9.32 \mathrm{E}-04$ & $9.37 \mathrm{E}-04$ & $9.32 \mathrm{E}-04$ & $9.37 \mathrm{E}-04$ & $9.32 \mathrm{E}-04$ \\
\hline $\mathrm{NO}_{3}$ & $1.60 \mathrm{E}-02$ & $1.59 \mathrm{E}-02$ & $1.60 \mathrm{E}-02$ & $1.59 \mathrm{E}-02$ & $1.60 \mathrm{E}-02$ & $1.59 \mathrm{E}-02$ & $1.60 \mathrm{E}-02$ & $1.59 \mathrm{E}-02$ & $1.60 \mathrm{E}-02$ & $1.59 \mathrm{E}-02$ \\
\hline $\mathrm{PO}_{4}$ & 1.37E-04 & $5.25 \mathrm{E}-10$ & $1.37 \mathrm{E}-04$ & $5.25 \mathrm{E}-10$ & $1.37 \mathrm{E}-04$ & $5.25 \mathrm{E}-10$ & $1.37 \mathrm{E}-04$ & $5.25 \mathrm{E}-10$ & $1.37 \mathrm{E}-04$ & $5.25 \mathrm{E}-10$ \\
\hline $\mathrm{SO}_{4}$ & $8.12 \mathrm{E}-05$ & 7.49E-05 & $8.12 \mathrm{E}-05$ & 7.49E-05 & $8.12 \mathrm{E}-05$ & 7.49E-05 & $8.12 \mathrm{E}-05$ & 7.49E-05 & $8.12 \mathrm{E}-05$ & $7.49 \mathrm{E}-05$ \\
\hline $\mathrm{Zn}$ & $8.19 \mathrm{E}-05$ & $9.55 \mathrm{E}-08$ & $8.19 \mathrm{E}-05$ & $9.55 \mathrm{E}-08$ & $8.19 \mathrm{E}-05$ & $9.55 \mathrm{E}-08$ & 8.19E-05 & $9.55 \mathrm{E}-08$ & $8.19 \mathrm{E}-05$ & $9.55 \mathrm{E}-08$ \\
\hline NTA & $1.00 \mathrm{E}-04$ & 8.37E-09 & $1.02 \mathrm{E}-04$ & 8.37E-09 & $1.00 \mathrm{E}-04$ & 8.37E-09 & $1.03 \mathrm{E}-04$ & 8.37E-09 & 1.10E-04 & 8.37E-09 \\
\hline MOPS & $1.00 \mathrm{E}-02$ & 4.31E-03 & $1.00 \mathrm{E}-02$ & $4.31 \mathrm{E}-03$ & $1.00 \mathrm{E}-02$ & 4.31E-03 & $1.00 \mathrm{E}-02$ & $4.31 \mathrm{E}-03$ & $1.00 \mathrm{E}-02$ & 4.31E-03 \\
\hline
\end{tabular}


Table S9: Percent recovery (\%) of total dissolved copper after exposure to C. reinhardtii in LZn, LMn, and LCo media.

\begin{tabular}{|c|c|c|c|c|c|c|c|c|c|}
\hline & \multicolumn{3}{|c|}{ LZn 1 to 4} & \multicolumn{3}{|c|}{ LMn 1 to 4} & \multicolumn{3}{|c|}{ LCo 1 to 4} \\
\hline & $\begin{array}{l}\mathrm{Cu}(\mathrm{g} / \mathrm{L}) \\
\text { before }\end{array}$ & $\begin{array}{c}\mathrm{Cu}(\mathrm{g} / \mathrm{L}) \\
\text { after }\end{array}$ & $\begin{array}{c}\text { Percent } \\
\text { recovery (\%) }\end{array}$ & $\begin{array}{l}\mathrm{Cu}(\mathrm{g} / \mathrm{L}) \\
\text { before }\end{array}$ & $\begin{array}{c}\mathrm{Cu}(\mathrm{g} / \mathrm{L}) \\
\text { after }\end{array}$ & $\begin{array}{c}\text { Percent } \\
\text { recovery } \\
\text { (\%) }\end{array}$ & $\begin{array}{l}\mathrm{Cu}(\mathrm{g} / \mathrm{L}) \\
\text { before }\end{array}$ & $\begin{array}{l}\mathrm{Cu}(\mathrm{g} / \mathrm{L}) \\
\text { after }\end{array}$ & $\begin{array}{c}\text { Percent } \\
\text { recovery } \\
(\%)\end{array}$ \\
\hline Cu1 & 4.95E-06 & $(4.17 \pm 0.17) \mathrm{E}-06$ & $84.3 \pm 3.5$ & & & & & & \\
\hline Cu2 & $1.71 \mathrm{E}-05$ & $(1.27 \pm 0.05) \mathrm{E}-05$ & $74.3 \pm 2.9$ & & & & & & \\
\hline Cu3 & $1.47 \mathrm{E}-04$ & $(1.46 \pm 0.03) \mathrm{E}-04$ & $99.3 \pm 1.7$ & $2.92 \mathrm{E}-05$ & $(1.73 \pm 0.10) \mathrm{E}-05$ & $59.2 \pm 3.4$ & $2.62 \mathrm{E}-05$ & $(1.93 \pm 0.05) \mathrm{E}-05$ & $73.6 \pm 2.0$ \\
\hline Cu4 & $1.43 \mathrm{E}-03$ & $(1.42 \pm 0.01) \mathrm{E}-03$ & $99.1 \pm 0.5$ & $2.71 \mathrm{E}-04$ & $(2.51 \pm 0.06) \mathrm{E}-04$ & $92.7 \pm 2.0$ & $2.62 \mathrm{E}-04$ & $(2.50 \pm 0.01) \mathrm{E}-04$ & $95.1 \pm 0.2$ \\
\hline
\end{tabular}


Table S10: Percent recovery (\%) of total dissolved copper after exposure to C. reinhardtii in LFe and HM media.

\begin{tabular}{l|ccc|ccc} 
& \multicolumn{3}{c}{ LFe 1 to 10 } & \multicolumn{3}{c}{ HM 1 to 10 } \\
\cline { 2 - 7 } & $\begin{array}{c}\text { Cu (g/L) } \\
\text { before }\end{array}$ & $\begin{array}{c}\text { Cu (g/L) } \\
\text { after }\end{array}$ & $\begin{array}{c}\text { Percent } \\
\text { recovery (\%) }\end{array}$ & $\begin{array}{c}\text { Cu (g/L) } \\
\text { before }\end{array}$ & $\begin{array}{c}\text { Cu (g/L) } \\
\text { after }\end{array}$ & $\begin{array}{c}\text { Percent } \\
\text { recovery (\%) }\end{array}$ \\
\hline Cu1 & $2.03 \mathrm{E}-05$ & $(1.35 \pm 0.48) \mathrm{E}-05$ & $66 \pm 24$ & $2.16 \mathrm{E}-06$ & $(3.4 \pm 1.7) \mathrm{E}-07$ & $15.6 \pm 8.1$ \\
Cu2 & $6.12 \mathrm{E}-05$ & $(5.34 \pm 0.94) \mathrm{E}-05$ & $87 \pm 15$ & $6.27 \mathrm{E}-06$ & $(3.8 \pm 1.5) \mathrm{E}-06$ & $61 \pm 24$ \\
Cu3 & $1.27 \mathrm{E}-04$ & $(1.05 \pm 0.30) \mathrm{E}-04$ & $82 \pm 24$ & $1.78 \mathrm{E}-05$ & $(1.45 \pm 0.03) \mathrm{E}-05$ & $81.4 \pm 1.9$ \\
Cu4 & $1.73 \mathrm{E}-04$ & $(1.49 \pm 0.25) \mathrm{E}-04$ & $86 \pm 15$ & $1.85 \mathrm{E}-04$ & $(1.59 \pm 0.01) \mathrm{E}-04$ & $85.8 \pm 0.4$ \\
Cu5 & $2.30 \mathrm{E}-04$ & $(2.24 \pm 0.32) \mathrm{E}-04$ & $97 \pm 14$ & $1.73 \mathrm{E}-03$ & $(1.54 \pm 0.01) \mathrm{E}-03$ & $89.4 \pm 0.8$ \\
Cu6 & $8.64 \mathrm{E}-04$ & $(8.47 \pm 0.36) \mathrm{E}-04$ & $98 \pm 42$ & $1.86 \mathrm{E}-02$ & $(1.47 \pm 0.01) \mathrm{E}-02$ & $78.9 \pm 0.6$ \\
Cu7 & $1.45 \mathrm{E}-03$ & $(1.43 \pm 0.34) \mathrm{E}-03$ & $98 \pm 24$ & $2.64 \mathrm{E}-02$ & $(1.91 \pm 0.04) \mathrm{E}-02$ & $72.3 \pm 1.3$ \\
Cu8 & $1.73 \mathrm{E}-03$ & $(1.72 \pm 0.18) \mathrm{E}-03$ & $100 \pm 10$ & $4.27 \mathrm{E}-02$ & $(3.46 \pm 0.05) \mathrm{E}-02$ & $81.1 \pm 1.1$ \\
Cu9 & $2.29 \mathrm{E}-03$ & $(2.25 \pm 0.31) \mathrm{E}-03$ & $98 \pm 14$ & $5.68 \mathrm{E}-02$ & $(4.80 \pm 0.05) \mathrm{E}-02$ & $84.4 \pm 0.8$ \\
Cu10 & $6.28 \mathrm{E}-03$ & $(6.37 \pm 0.21) \mathrm{E}-03$ & $101 \pm 3$ & $1.58 \mathrm{E}-01$ & $(1.32 \pm 0.01) \mathrm{E}-01$ & $83.2 \pm 0.8$ \\
Average & & & & & & \\
recovery & & & $\mathbf{9 2 \pm 1 8}$ & & & $\mathbf{7 3 . 3 \pm 4 . 0}$ \\
\hline
\end{tabular}


Table S11: Percent recovery (\%) of copper after acid digestion of IAEA-413 algae material.

\begin{tabular}{ccc}
\hline $\begin{array}{c}\text { Mesured } \\
\text { concentrations } \\
\left(\mathbf{m g} \cdot \mathbf{k g}^{-1}\right)\end{array}$ & $\begin{array}{c}\text { Expected } \\
\text { concentrations } \\
\left(\mathbf{m g} \cdot \mathbf{k g}^{-1}\right)\end{array}$ & $\begin{array}{c}\text { Percent } \\
\text { recovery }(\%)\end{array}$ \\
\hline 10.8 & $\mathbf{1 1 . 1}$ & 97.3 \\
10.3 & $\mathbf{1 1 . 1}$ & 93.1 \\
10.8 & $\mathbf{1 1 . 1}$ & 96.9 \\
11.2 & $\mathbf{1 1 . 1}$ & 100.9 \\
10.6 & $\mathbf{1 1 . 1}$ & 95.3 \\
10.5 & $\mathbf{1 1 . 1}$ & 94.6 \\
10.7 & $\mathbf{1 1 . 1}$ & 96.5 \\
9.86 & $\mathbf{1 1 . 1}$ & 88.8 \\
Average recovery & & $\mathbf{9 5 . 4} \pm \mathbf{3 . 5}$ \\
\hline
\end{tabular}


Table S12: Summary of free $\mathrm{Cu}^{2+}$ toxicity data found in the literature.

\begin{tabular}{|c|c|c|c|c|}
\hline Species & $\mathrm{pH}$ & Endpoint & EC50 & Ref \\
\hline C. reinhardtii & 6.8 & 120-h growth & $400 \mathrm{nM} \mathrm{Cu}^{2+}(\mathrm{IC} 30)$ & 1 \\
\hline $\begin{array}{l}\text { Scenedesmus } \\
\text { quadricauda }\end{array}$ & $5.0-8.5$ & $\begin{array}{l}\text { Inhibition of } \\
\text { phosphorus uptake } \\
(<1 \mathrm{~h})\end{array}$ & $0.076-8320 \mathrm{nM} \mathrm{Cu}^{2+}$ & 2 \\
\hline P. subcapitata & 8.1 & ?-h growth & $12 \mathrm{nM} \mathrm{Cu}^{2+}$ & 3 \\
\hline $\begin{array}{l}\text { P. subcapitata } \\
\text { Chlorella sp. }\end{array}$ & 7.9 & 72-h growth & $\begin{array}{l}47 \mathrm{nM} \mathrm{Cu}_{\text {tot }}(\sim 30 \% \text { free }) \\
30 \mathrm{nM} \mathrm{Cu}_{\text {tot }}(\sim 30 \% \text { free })\end{array}$ & 4 \\
\hline C. reinhardtii & 7.5 & 24-h growth & $6.0 \mathrm{nM} \mathrm{Cu}^{2+}$ & 5 \\
\hline C. reinhardtii & 7.5 & 24-h growth & $7 \mathrm{nM} \mathrm{Cu}^{2+}$ & 6 \\
\hline $\begin{array}{l}\text { Scenedesmus } \\
\text { acuminatus }\end{array}$ & 6.8 & 96-h growth & $33 \mu \mathrm{M} \mathrm{Cu}^{2+}$ & 7 \\
\hline Chlorella sp. & $5.5-8.0$ & 48-h growth & $11-275 \mathrm{nM} \mathrm{Cu}^{2+}$ & 8 \\
\hline P. subcapitata & $5.7-8.6$ & 72-h growth & $0.040-430 \mathrm{nM} \mathrm{Cu}^{2+}$ & 9 \\
\hline $\begin{array}{l}\text { P. subcapitata } \\
\text { C. vulgaris }\end{array}$ & $5.5-8.7$ & 72-h growth & $\begin{array}{l}0.039-347 \mathrm{nM} \mathrm{Cu}^{2+} \\
0.26-3240 \mathrm{nM} \mathrm{Cu}^{2+}\end{array}$ & 10 \\
\hline $\begin{array}{l}\text { P. subcapitata } \\
\text { Chlorella sp. }\end{array}$ & $\begin{array}{l}5.9-8.5 \\
6-7.5\end{array}$ & $\begin{array}{l}\text { 72-h growth } \\
\text { 48-h growth }\end{array}$ & $\begin{array}{l}0.56-470 \mathrm{nM} \mathrm{Cu}^{2+} \\
2.8-330 \mathrm{nM} \mathrm{Cu}^{2+}\end{array}$ & 11 \\
\hline $\begin{array}{l}\text { P. subcapitata } \\
\text { Chlorella sp. }\end{array}$ & 7.5 & 72-h growth & $\begin{array}{l}52-130 \mathrm{nM} \mathrm{Cu}^{2+} \\
36-130 \mathrm{nM} \mathrm{Cu}^{2+}\end{array}$ & 12 \\
\hline $\begin{array}{l}\text { Navicula } \\
\text { pelliculosa }\end{array}$ & 7.0 & 72-h growth & $420-540 \mathrm{nM} \mathrm{Cu}^{2+}$ & 13 \\
\hline Chlorella sp. & $5.7-6.5$ & 72-h growth & $21-550 \mathrm{nM} \mathrm{Cu}^{2+}$ & 14 \\
\hline C. reinhardtii & 7.0 & 72-h growth & $0.0042-2.3 \mathrm{nM} \mathrm{Cu}^{2+}$ & This study \\
\hline
\end{tabular}



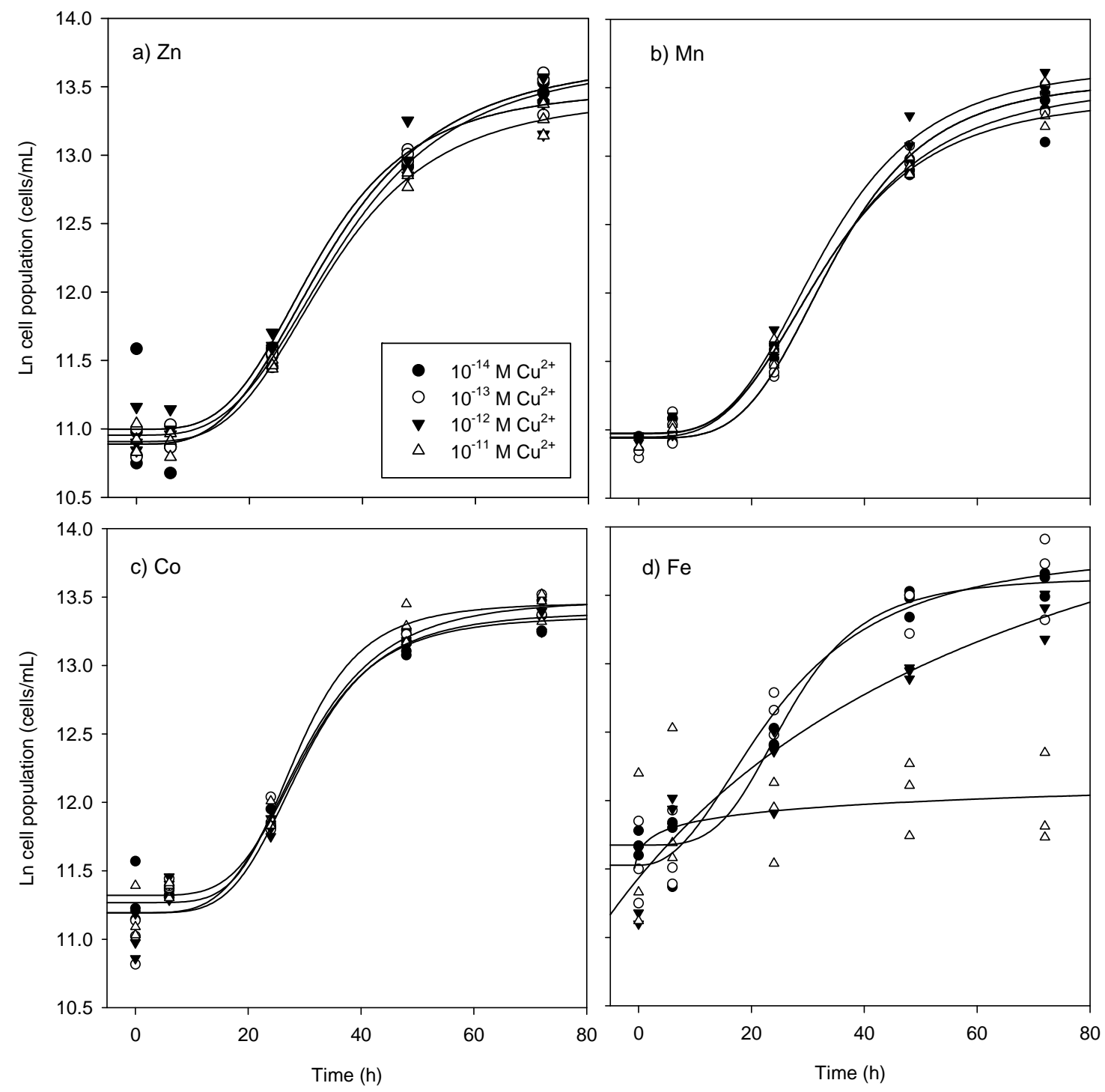

Figure S1: Cellular growth over time of C. reinhardtii exposed to four different concentrations of free copper $\left(10^{-14}, 10^{-13}, 10^{-12}\right.$ and $\left.10^{-11} \mathrm{M}\right)$ in four low-concentrated media in $\mathrm{Zn}$ (a), Mn (b), Co (c) and $\mathrm{Fe}(\mathrm{d})$. 


\section{References}

1. Macfie, S. M.; Tarmohamed, Y.; Welbourn, P. M., Effects of cadmium, cobalt, copper, and nickel on growth of the green alga Chlamydomonas reinhardtii: The influences of the cell wall and $\mathrm{pH}$. Arch Environ Contam Toxicol 1994, 27, 454-458.

2. Peterson, H. G.; Healey, F. P.; Wagemann, R., Metal toxicity to algae - a highly pH dependent phenomenon. Can J Fish Aquat Sci 1984, 41, (6), 974-979.

3. Nagai, T.; Kamo, M., Comparative modeling of the effect of metal mixtures on algal growth using biotic ligand model, concentration additive, and independent action. Jpn J Environ Toxicol 2014, 17, (2), 57-68.

4. Angel, B. M.; Simpson, S. L.; Granger, E.; Goodwyn, K.; Jolley, D. F., Time-averaged concentrations are effective for predicting chronic toxicity of varying copper pulse exposures for two freshwater green algae species. Environ Pollut 2017, 230, 787-797.

5. Stoiber, T. L.; Shafer, M. M.; Armstrong, D. E., Relationships between surface-bound and internalized copper and cadmium and toxicity in Chlamydomonas reinhardtii. Environ Toxicol Chem 2012, 31, (2), 324-335.

6. Stoiber, T. L.; Shafer, M. M.; Armstrong, D. E., Differential effects of copper and cadmium exposure on toxicity endpoints and gene expression in Chlamydomonas reinhardtii. Environ Toxicol Chem 2010, 29, (1), 191-200.

7. Lombardi, A. T.; Hidalgo, T. M. D.; Vieira, A. A. H.; Sartori, A. L., Toxicity of ionic copper to the freshwater microalga Scenedesmus acuminatus (Chlorophyceae, Chlorococcales). Phycologia 2007, 46, (1), 74-78.

8. Wilde, K. L.; Stauber, J. L.; Markich, S. J.; Franklin, N. M.; Brown, P. L., The effect of pH on the uptake and toxicity of copper and zinc in a tropical freshwater alga (Chlorella sp.). Arch Environ Contam Toxicol 2006, 51, (2), 174-185.

9. De Schamphelaere, K. A. C.; Vasconcelos, F. M.; Heijerick, D. G.; Tack, F. M. G.; Delbeke, K.; Allen, H. E.; Janssen, C. R., Development and field validation of a predictive copper toxicity model for the green alga Pseudokirchneriella subcapitata. Environ Toxicol Chem 2003, 22, (10), 2454-2465.

10. De Schamphelaere, K. A. C.; Janssen, C. R., Bioavailability models for predicting copper toxicity to freshwater green microalgae as a function of water chemistry. Environ Sci Technol 2006, 40, (14), 4514-4522.

11. De Schamphelaere, K. A. C.; Stauber, J. L.; Wilde, K. L.; Markich, S. J.; Brown, P. L.; Franklin, N. M.; Creighton, N. M.; Janssen, C. R., Toward a biotic ligand model for freshwater green algae: Surface-bound and internal copper are better predictors of toxicity than free $\mathrm{Cu}^{2+}$-ion activity when $\mathrm{pH}$ is varied. Environ Sci Technol 2005, 39, (7), 2067-2072.

12. Franklin, N. M.; Stauber, J. L.; Apte, S. C.; Lim, R. P., Effect of initial cell density on the bioavailability and toxicity of copper in microalgal bioassays. Environ Toxicol Chem 2002, 21, (4), 742-751.

13. Nagai, T.; De Schamphelaere, K. A., The effect of binary mixtures of zinc, copper, cadmium, and nickel on the growth of the freshwater diatom Navicula pelliculosa and comparison with mixture toxicity model predictions. Environ Toxicol Chem 2016, 35, (11), 2765-2773.

14. Franklin, N. M.; Stauber, J. L.; Markich, S. J.; Lim, R. P., pH-dependent toxicity of copper and uranium to a tropical freshwater alga (Chlorella sp.). Aquat Toxicol 2000, 48, (2-3), 275289. 